\title{
Essais
}

Revue interdisciplinaire d'Humanités

Hors-série 7 | 2022

Récits d'outre-thèse

\section{Docteures en situation de handicap : regards et enjeux}

Women doctors with disabilities: Perspectives and issues

Anaïs Carrere et Maria Gabriela Dascalakis-Labreze

\section{CpenEdition}

Journals

Édition électronique

URL : https://journals.openedition.org/essais/10833

DOI : $10.4000 /$ essais. 10833

ISSN : 2276-0970

Éditeur

École doctorale Montaigne Humanités

Édition imprimée

Date de publication : 15 avril 2022

ISBN : 979-10-970024-00-0

ISSN : 2417-4211

Référence électronique

Anaïs Carrere et Maria Gabriela Dascalakis-Labreze, « Docteures en situation de handicap : regards et enjeux », Essais [En ligne], Hors-série 7 | 2022, mis en ligne le 01 février 2022, consulté le 18 janvier 2023. URL : http://journals.openedition.org/essais/10833 ; DOI : https://doi.org/10.4000/essais. 10833

Ce document a été généré automatiquement le 18 janvier 2023.

Tous droits réservés 


\title{
Docteures en situation de handicap : regards et enjeux
}

\author{
Women doctors with disabilities: Perspectives and issues
}

Anaïs Carrere et Maria Gabriela Dascalakis-Labreze

\section{Introduction}

1 Notre rencontre a eu lieu pour la première fois il y a quatre ans dans le transport d'accompagnement ${ }^{1}$ qui nous amène à l'Université Bordeaux Montaigne. Nous nous croisions au moins deux fois par semaine et partagions les mêmes questionnements et motivations. D'une semaine à l'autre, les conversations tournaient autour de nos thèses, de nos enseignements et de temps en temps des démarches à faire par rapport à nos handicaps, devant l'oreille attentive de notre chauffeur, Monsieur Meynard, qui assistait aux débats sur le président américain, sur la politique française en Argentine ou sur des formulaires à remplir avec le même intérêt. Puisque c'est le handicap qui nous a réunies, nous tenions à rendre visibles nos parcours de femmes en situation de handicap enseignantes et chercheuses, conscientes du fait que nous ne sommes pas forcément représentées dans l'Enseignement supérieur. Plusieurs questions en lien intrinsèque avec nos situations personnelles qui influent aussi sur le professionnel se posent dont celle de l'insertion des personnes en situation de handicap (visibles ou invisibles) dans le milieu de la Recherche et de l'Enseignement supérieur ces dernières années. Que révèlent les chiffres? Le graphique ci-dessous montre que seulement $0,8 \%$ des personnes en situation de handicap accèdent aux études dites de troisième cycle en doctorat et nourrissent l'espoir d'une potentielle insertion professionnelle ${ }^{2}$ dans le milieu universitaire. 
Fig. 1. Évolution de la répartition des étudiants en situation de handicap à l'Université par cycle ${ }^{3}$

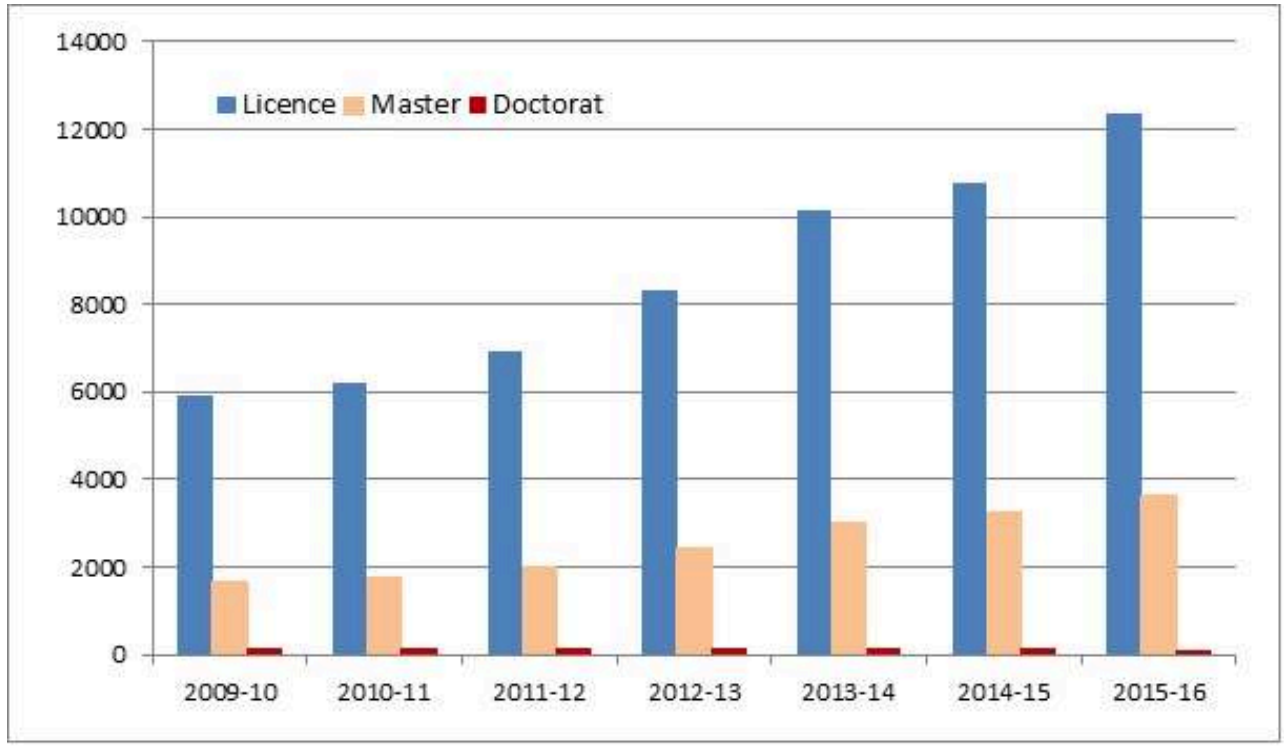

Le chemin de thèse est un sentier singulier qui peut très vite décourager ou passionner. Face à la page blanche, nous sommes tous et toutes à égalité. Nos situations personnelles peuvent peu ou prou perturber la trajectoire de nos travaux. Nous souhaitons donc partager avec vous nos aventures respectives de thèse. Tout d'abord, une première voie nous conduira à découvrir le parcours d'Anaïs Carrere dont la soutenance de thèse a eu lieu en septembre 2021. Puis, nous partirons à la rencontre de Maria Gabriela Dascalakis-Labreze, docteure depuis novembre 2018. Il s'agira, dans les deux cas, de découvrir nos parcours de recherche et nos situations singulières. Avant de conclure, nous vous présenterons de manière croisée nos perspectives d'avenir foncièrement liées à nos conditions respectives.

\section{Anaïs Carrere}

3 En septembre 2016, j'ai eu le privilège d'obtenir un contrat doctoral "fléché handicap $»^{4}$ qui permet aux doctorants en situation de handicap d'enseigner à l'Université tout en menant leurs recherches financées pendant trois ans, avec possibilité d'une quatrième année dérogatoire. Cette opportunité m'a permis de m'immerger dans le milieu universitaire. Chargée de cours ${ }^{5}$ en linguistique anglaise au sein du Département Études des Mondes Anglophones à l'Université Bordeaux Montaigne, j'appartiens au laboratoire CLIMAS ${ }^{6}$. Ma thèse porte sur la "Contribution des Language and Gender Studies à l'analyse du discours de femmes engagées: de la mise en perspective historique à la validation empirique». En parallèle, se vit une aventure personnelle qui ne saurait être tue dans ce récit : je suis porteuse d'un handicap moteur qui me contraint au quotidien à me déplacer en fauteuil roulant manuel, équipé du système motorisé Minotor. Ce type de fauteuil me permet d'accéder plus facilement à des endroits habituellement inatteignables.

4 En fin de Master 2 recherche, j'ai eu envie de débuter une thèse doctorale. Lors d'un séminaire sur la pragmatique du discours, j'ai découvert une interview de la linguiste Deborah Tannen qui expliquait de façon très concrète les concepts de styles conversationnels et interactionnels différenciés qu'elle a élaborés au milieu des 
années 1980. Dans cette vidéo, deux garçons interagissent de manière vive, se chamaillent, se donnent des coups, puis deux petites filles discutent, partagent, on ne sait quel secret ou potin... Je me souviens avoir ri et immédiatement pensé : « c'est moi avec mes sœurs, ma maman, mes amis lors de repas et de pauses goûters enflammés ". De là est né le souhait de travailler sur le langage, les comportements linguistiques et interactionnels genrés. Mes différentes lectures sur le sujet m'ont permis de comprendre qu'il était certes théorique mais demeurait actuel, suscitant même un certain nombre d'émotions et de réactions positives : le rire. Ce sujet cache, néanmoins, des réalités implicites : le contrôle des productions, des représentations du discours, des êtres humains, de leur identité à la fois collective et individuelle de façon bien souvent inégale. Ma thèse retrace l'émergence des Language and Gender Studies dans les pays anglophones, en caractérise l'épistémologie et éprouve l'applicabilité dans le domaine du discours politique. Discipline hybride, à la croisée des études de genre et de la linguistique générale, l'étude linguistique des rapports de genre au sein des sociétés reste largement méconnue du grand public et de façon plus surprenante encore, semble ignorée de beaucoup d'universitaires. Dans les trois premiers volets de mon travail, sont apportés un cadrage contextuel et une description analytique des grands courants historiques qui structurent cette discipline depuis sa fondation dans les années 1970. Le dernier chapitre est consacré à une mise à l'épreuve du cadre théorique et méthodologique à travers l'analyse concrète du discours politique oral d'Hillary Clinton (2007-2016) et de Donald Trump (2015-2016). Il s'agit de déterminer dans quelle mesure les formes instituées de l'expression langagière sont à la fois révélatrices et instigatrices de la construction sociocognitive du genre et du pouvoir. Hillary Clinton s'avère victime du principe d'injonction paradoxale, la double-contrainte, qui frappe les femmes en politique ${ }^{7}$. Cette dure réalité avait été perçue et dénoncée par la linguiste féministe Robin Lakoff dès 1975. J'en évalue les conséquences sur l'identité personnelle et politique d'Hillary Clinton. Cette étude de cas nous plonge au cœur du langage dans ses deux principales fonctions: idéationnelle et interactionnelle ${ }^{8}$ qui constituent d'ailleurs un des fils conducteurs de ma réflexion. Le langage permet en effet aux humains de construire, d'institutionnaliser et de diffuser dans les esprits de toute une communauté un ensemble d'idées et de catégories liées à l'expérience humaine. Les représentations de l'expérience qui en découlent sont favorables aux dominants, à ceux qui historiquement contrôlent le système expressif 9 .

$5 \mathrm{Au}$ cours de cette aventure de thèse, il n'a pas été évident de traiter de la notion de genre car beaucoup de disciplines et de nombreux théoriciens français et anglophones ont des points de vue divergents et parfois contradictoires suivant les époques et évolutions de la recherche et des mentalités sur le sujet. Le thème du genre reste controversé, fragile, suscitant des polémiques, des divergences d'appropriation, à la croisée du terrain militant, des minorités et de la théorisation universitaire. C'est, en effet, un sujet fondamentalement politique qui demeure en tension. Pour ce qui est de la recherche de terrain, construire un cadre méthodologique clair respectant scrupuleusement les normes méthodologiques de recherche, en vigueur en Sciences humaines et sociales, demeure un enjeu difficile auquel aucun chercheur n'est réellement préparé en amont de la thèse. Bien souvent, il découvre au cours du parcours doctoral, les injonctions administratives imposées par la mise en place d'une enquête, d'un questionnaire ou dans la direction d'entretiens dirigés ou semi-dirigés. Plusieurs tâches constituent une charge mentale: la rédaction du formulaire d'acceptation des participants, le respect de la protection des données de chaque 
participant, ou encore les charges administratives à anticiper au moins six mois à l'avance lorsqu'il faut se déplacer dans une autre région de France ou l'étranger pour mener à bien ses recherches tels que les documents de charges de missions, la sollicitation de l'École Doctorale ou des laboratoires pour obtenir une participation financière liée aux divers frais de déplacements.

\section{Maria Gabriela Dascalakis-Labreze}

Docteure en langues et littératures étrangères depuis novembre 2018, je me suis inscrite en doctorat à l'Université de Pau et des Pays de l'Adour pour mener à bien un travail de recherche intitulé «La reconfiguration des faits dans le discours de presse argentine. Les visites des présidents français Charles De Gaulle (1964), François Mitterrand (1987) et Jacques Chirac (1997) $»^{10}$. J'ai fait ma thèse doctorale tout en travaillant à plein temps en tant que $\mathrm{PRCE}^{11}$ de français langue étrangère au Département de Français langue étrangère ${ }^{12}$ de l'Université Bordeaux Montaigne. Je suis également maman d'une petite fille de 9 ans qui avait un an au début de ma thèse. Atteinte d'un handicap visuel congénital, j'ai dû réadapter ma recherche à mon état de santé qui s'est aggravé pendant mon parcours doctoral. J'ai pu surmonter ces obstacles grâce aux aides techniques mises à disposition lors de l'aménagement de mon poste de travail : un télé-agrandisseur qui me permet d'agrandir les textes à lire, un scanner et un logiciel à commandes vocales m'aidant à écrire, à lire et pouvant même lire à ma place.

7 L'idée de travailler sur la visite des présidents français en Argentine est née en 2003 lorsque j'habitais encore dans mon pays natal, l'Argentine, et que j'ai appris que le président Charles De Gaulle s'était rendu sur le sol du pays en 1964. Ayant constaté que cet événement ne faisait que peu de lignes dans les livres d'Histoire, j'ai plongé mon regard dans la presse nationale argentine pour me renseigner et réaliser une analyse exploratoire. Arrivée en France quelques années plus tard, j'ai ressenti le besoin d'élargir ces bribes de réflexion débutée en Argentine et de les concrétiser dans une thèse doctorale. Il fallait donc déterminer un sujet et un objet de recherche. J'ai décidé d'interroger les visites des trois présidents français s'étant rendus en Argentine au $\mathrm{XX}^{\mathrm{e}}$ siècle Charles De Gaulle, François Mitterrand et Jacques Chirac et de retracer le retentissement de leurs venues dans la presse argentine. Parallèlement à la définition de mon sujet, j'ai décidé de travailler sur cinq journaux nationaux argentins Clarín, Crónica, La Nación, La Prensa et La Razón offrant un éventail assez large de lignes éditoriales. Je me suis proposée d'interroger la construction de ces trois événements programmés dans les quotidiens mentionnés afin de repérer l'influence française dans les discours politiques et médiatiques argentins (francophilie des transferts) et de déterminer comment les journaux les présentent en fonction de leur «identité discursive $»^{13}$. Cette idée aurait difficilement vu le jour sans mon installation en France car, loin du pays, je n'arrivais plus à saisir les «faits » lorsque je lisais mon journal de prédilection. Une forte sensation de décalage entre le fait et sa mise en récit s'étant installée, j'ai commencé à multiplier mes lectures et à cerner des disparités. L'éloignement géographique était en train d'œuvrer et me permettait de mieux mettre à distance les tensions politiques qui sous-tendent le discours de presse argentine. Pour bien déceler les récits sous-jacents, il fallait replacer les visites dans leurs contextes historiques respectifs aussi bien en France qu'en Argentine. Dans la construction de ces 
événements, les récits des trois visites mettent en lumière la fascination argentine pour la France et le transfert du mythe de la grandeur française vers la nation argentine, outre les revendications politiques intérieures très marquées.

8 Au début de mon parcours de recherche, le plus déroutant a été le cheminement pour découvrir une méthodologie, une approche et adopter un cadre théorique. Avant l'aventure, j'imaginais un chapitre théorique prêt-à-déguster que je pourrais réchauffer et appliquer sans problèmes à mon corpus. Hélas ! J'ai dû emprunter le chemin inverse. Je me suis attelée à définir les frontières du corpus afin de le questionner en appliquant une matrice temporelle commune aux trois visites et aux cinq journaux. Or, les conditions matérielles du recueil, de la mise en forme, du classement et de l'analyse du corpus n'ont pas été de tout repos à cause du mauvais état de conservation des journaux et de leur mise à disposition dans les archives ${ }^{14}$. En outre, si mon téléagrandisseur et le logiciel basse vision m'ont vraiment aidée à travailler sur le corpus, la tâche s'est avérée ardue car ces compensations techniques nécessitent des efforts visuels supplémentaires. En effet, l'utilisation du logiciel d'agrandissement offre une vision parcellaire des documents, ce qui ralentit leur aperçu et leur compréhension d'ensemble. Par ailleurs, le choix du cadre théorique n'a pas été chose aisée car il fallait maitriser plusieurs champs disciplinaires tels que les Sciences de l'information et de la communication, l'analyse du discours, l'Histoire, entre autres. J'ai donc réorienté mes lectures théoriques et me suis proposée de dépasser le cadre strictement verbal pour embrasser la multiplicité de signes investis dans la page du journal en vue de repérer leur enchâssement et les sens qui en découlaient. Plusieurs lectures ont jalonné cette réflexion, mais c'est sans aucun doute l'article sur «l'aire scripturale $»^{15}$ qui m'a éclairée et m'a amenée à repenser mon travail depuis une approche sémio-discursive. Dans ce sens, certains travaux sur l'événement et la presse ${ }^{16}$ et sur les récits ${ }^{17}$ m'ont permis de réfléchir sur les mécanismes de production de sens aussi bien dans la presse que dans les discours politiques. Les résultats de ma thèse montrent que les visites officielles des présidents sont des événements programmés dont le récit engendre de nouveaux événements. Ce phénomène que j’ai appelé le "glissement événementiel» révèle que les voyages des présidents français en Argentine agissent comme un déclencheur des récits politisés qui envahissent la page du journal, à travers différentes stratégies narratives, reléguant l'événement principal au second plan. Ainsi, l'événement-visite laisse émerger un nouvel événement ancré dans la problématique de politique intérieure argentine propre à chaque période. Le glissement événementiel, tel un processus collatéral, réussit à installer avec plus ou moins de force un nouvel événement qui s'impose et semble même remettre en question le sens des voyages présidentiels. Son intérêt ultime est l'hypothèse qui ouvre la voie à d'autres travaux concernant les récits sous-jacents induits selon les lignes éditoriales des journaux transposables à d'autres contextes politiques et médiatiques.

Après avoir retracé nos chemins de recherche et décrit nos singularités, nous allons croiser nos regards sur deux points essentiels de nos parcours, notamment l'accessibilité et l'insertion professionnelle post-doctorale.

\section{Récits croisés d'avenir}

9 L'expérience de tout chercheur débute par une longue phase de découverte de ses objets de recherche à travers la lecture et l'exploration de sources primaires et 
secondaires en lien avec le sujet. Pour ce faire, il est bien souvent nécessaire de se rendre dans les bibliothèques universitaires spécialisées. Nos lecteurs pourraient estimer que se rendre à la bibliothèque pour emprunter des ouvrages est une entreprise anodine. Si la loi prévoit une accessibilité dite "universelle ${ }^{18}$ aux bâtiments publics et aux aménagements, lorsque nous sommes en situation de handicap, nous constatons qu'une disparité subsiste sur le terrain. Ainsi, circuler aisément en fauteuil roulant dans les rayons de bibliothèque pour attraper un ouvrage ou essayer de repérer la cote sur une étagère située en hauteur constituent de véritables défis. Il est donc souvent nécessaire de faire appel à des aides humaines pour accomplir ces missions. Même si l'Université procède à une compensation juste de toutes les situations de handicap, il n'en demeure pas moins vrai que nous devons toujours tout anticiper que ce soit en termes de déplacement et d'aménagement de notre lieu de travail. Par conséquent, une charge mentale supplémentaire est provoquée par nos situations singulières.

Comme pour beaucoup de jeunes chercheurs, la problématique de l'insertion professionnelle au sein de l'Université se pose pour nous après la soutenance de la thèse. Cette source d'inquiétude est d'autant plus forte qu'on connaît la pénurie des postes de MCF dans le supérieur et que nos situations de handicap ne nous permettent pas forcément d'avancer dans nos recherches au même rythme que nos collègues. Nous sommes confrontées à deux situations statutaires différentes, mais fondées sur la même problématique : nous ne pouvons pas postuler ailleurs, en France, aux postes de MCF étant donné les contraintes induites pas nos handicaps. Ainsi, Anaïs pourrait candidater à un éventuel poste de MCF fléché handicap dans son domaine de recherche à condition qu'il y ait ouverture de poste. Le cas de Gabriela témoigne d'un autre handicap, en tant que PRCE fonctionnaire, il est impossible de candidater à un poste fléché handicap, réservé aux enseignants contractuels, non fonctionnaires ${ }^{19}$. Elle pourrait éventuellement y accéder lors d'une promotion interne ${ }^{20}$ de l'établissement. Nos deux cas de figure montrent que l'insertion professionnelle des docteures en situation de handicap dans la recherche n'est pas une évidence, contrairement aux idées reçues. Ces perspectives nébuleuses d'avenir ne nous empêchent pas pour autant de nous engager pleinement dans notre passion pour l'enseignement et la recherche et nous poussent à continuer à publier, à communiquer et à poursuivre la mission de vulgarisation de nos travaux ${ }^{21}$.

\section{Conclusions}

11 Par ce récit croisé de docteures, nous souhaitions évoquer nos situations de handicap respectives, visible, invisible, souvent méconnues, mais qui touchent dans l'ombre, certains doctorants et enseignants-chercheurs. Bien que les situations décrites incarnent un certain nombre d'obstacles patents et communs à tous les parcours doctoraux, elles se veulent porteuses d'espoir. Nos années d'études doctorales ont été marquées par des événements d'ordre divers, mais elles ont surtout été jalonnées par la curiosité. Faire une thèse n'est pas une mince affaire, elle l'est d'autant moins quand on est femme, étrangère, mère, que l'on travaille à plein temps et que l'on est, de surplus, en situation de handicap. En cela, le parcours doctoral s'apparente beaucoup à celui des personnes en situation de handicap qui, pour avancer, doivent s'adapter sans cesse à de nouvelles situations. Cette capacité d'adaptation et de réadaptation est la clé de voûte 
de toute recherche et constitue vraisemblablement l'une des compétences majeures des docteurs et des doctorants. $\mathrm{Si}$ «[p]ar définition, les déficiences n'excluent pas du travail ; toutefois, les conditions sociales d'usage des corps et la nature des emplois rendent cette rencontre statistiquement difficile $»^{22}$, nous espérons alors que nos récits permettront une réflexion profonde sur la nécessité d'une ouverture plus large du monde académique vers l'insertion, le maintien dans l'emploi, mais également la promotion des docteurs en situation de handicap, contractuels ou fonctionnaires.

\section{NOTES}

1. En tant que personnel en situation de handicap, nous avons des postes aménagés et un transport domicile-travail qui assure nos déplacements. Nous remercions Violaine Lafourcade et Pauline Pinto du Pôle Handicap de l'Université Bordeaux Montaigne pour leur accompagnement personnalisé.

2. Après la soutenance de la thèse, les personnes en situation de handicap ont deux voies pour devenir maîtresses de conférences selon leur statut. Les docteurs contractuels peuvent postuler à un poste fléché handicap. Or, pour les docteurs en situation de handicap déjà fonctionnaires la situation est bien plus complexe car leur recrutement se fait par la voie classique, ce qui diminue fortement les chances d'être recrutés.

3. Isabelle Kabla-Langlois (coord.), L'état de l'Enseignement supérieur de la Recherche en France. Paris, Ministère de l'Enseignement supérieur, de la Recherche et de l'Innovation, 2017, p. 41. Source : MENESR-DGESIP. https://publication.enseignementsup-recherche.gouv.fr/eesr/10/l-etat-de-lenseignement-superieur-et-de-la-recherche-en-france-10.php.

4. La campagne "doctorat handicap " propose chaque année, un financement de 25 contrats à des étudiants présentant un projet de thèse et reconnus bénéficiaires de l'obligation d'emploi (BOE).

5. Le service d'enseignement d'un chargé de cours et d'un doctorant contractuel affecté dans le supérieur s'élève à 64 heures annuelles.

6. Je suis titulaire d'une licence en Langues, littératures et civilisations étrangères et régionales et d'un Master 2 recherche en études anglophones de l'Université Bordeaux Montaigne.

7. Ce concept est résumé ainsi : «Damned if you do; damned if you don't [go by the conversational rules assigned to you as a woman] ", Robin Lakoff, Language and Woman's Place: Text and Commentaries. Revised and expanded edition, Oxford New York, Oxford University Press, [1975] 2004, p. 89.

8. Nicole Delbecque, Linguistique cognitive. Comprendre comment fonctionne le langage, Bruxelles, De Boeck, 2002.

9. Dale Spender, Man made language. First Edition, United Kingdom, Pandora Press, 1980.

10. Disponible sur http://www.theses.fr/2018PAUU1044.

11. Professeure certifiée ayant passé le CAPES externe d'espagnol. Le service d'enseignement d'un professeur du secondaire affecté dans le supérieur s'élève à 384 heures annuelles.

12. J'y ai été recrutée en 2012. Argentine d'origine, titulaire d'un Master 2 recherche en langue et littérature françaises et d'un Master 2 enseignement en langue français de la Faculté des langues de l'Université nationale de Cordoba, je me suis installée en France après avoir travaillé pendant plusieurs années en Argentine.

13. Jean Pierre Esquenazi, L'écriture de l'actualité, Grenoble, PUG, 2013, p. 138. 
14. Je me suis rendue trois fois dans les archives à Buenos Aires, sans financement particulier.

15. Jean Peytard, «Lecture(s) d'une "aire scripturale" : la page de journal », Langue française. Textes et discours non littéraires, 28, 1975, p. 39-59. DOI : https://doi.org/10.3406/lfr.1975.6090.

16. Joceyline Arquembourg, L'événement et les médias. Les récits médiatiques des tsunamis et les débats publics (1755-2004), Paris, Édition des Archives Contemporaines, 2011. Lucrecia Escudero Chauvel, Malvinas, el gran relato. Fuentes y rumores en la información de guerra, Barcelona, Gedisa. Colección el mamífero parlante, 1996. Eliséo Véron, Construire l'événement. Les médias et l'accident de Three Mile Island, Paris, Les Éditions de Minuit, 1981.

17. Dardo Scavino, Rebeldes y confabulados. Narraciones de la política argentina, Buenos Aires, Eterna Cadencia, 2012.

18. https://handicap.gouv.fr/vivre-avec-un-handicap/handicap-accessibilite-et-deplacement/ article/accessibilite-universelle.

19. Revoir notre note $n^{\circ} 3$ sur le recrutement des personnes en situation de handicap.

20. En vertu de l'article 93 de la Loi de transformation de la fonction publique, il existe une possibilité de promotion ascensionnelle par la voie du détachement. Ce dispositif récent est actuellement à l'état d'expérimentation jusqu'en 2025.

21. En novembre 2020, nous avons participé à la "Nuit européenne des chercheur.e.s ", un évènement annuel, par le biais de la diffusion d'un podcast sur le thème de : «Recherche et Handicap : les secrets des défis que nous relevons » en collaboration avec CAP SCIENCES, le CNRS et l'Université Bordeaux Montaigne. Disponible sur https://www.youtube.com/watch? v=UiMdgZT6Xy4.

22. Joël Zaffran, Accessibilité et handicap, Grenoble, Presses universitaires de Grenoble, 2015, p. 25-26.

\section{AUTEURS}

\section{ANAÏS CARRERE}

Laboratoire CLIMAS, Université Bordeaux Montaigne

MARIA GABRIELA DASCALAKIS-LABREZE

Laboratoire MICA, axe IDEM, Université Bordeaux Montaigne 